\title{
A Broadband Linear-to-Circular Transmission Polarizer Based on Right-Angled Frequency Selective Surfaces
}

\author{
Wei Zhang, Jian-ying Li, and Jian Xie \\ School of Electronics and Information, Northwestern Polytechnical University, Xi'an 710072, China \\ Correspondence should be addressed to Wei Zhang; zhangwei19900410@163.com
}

Received 7 August 2017; Revised 26 November 2017; Accepted 3 December 2017; Published 27 December 2017

Academic Editor: Francisco Falcone

Copyright (c) 2017 Wei Zhang et al. This is an open access article distributed under the Creative Commons Attribution License, which permits unrestricted use, distribution, and reproduction in any medium, provided the original work is properly cited.

\begin{abstract}
A broadband linear-to-circular transmission polarizer using a right-angled frequency selective surface is presented in this paper. The new proposed polarizer has both advantages of wide operational bandwidth and high transmission coefficients. To verify the design, a new polarizer working in X-band is optimized and fabricated. Experimental results show that the measured axial ratios lower than $3 \mathrm{~dB}$ range from 6.42 to $13.70 \mathrm{GHz}$, with low insertion loss. Meanwhile, the polarizer can operate over a broad range of frequencies from 6.75 to $10.75 \mathrm{GHz}$, when the incident angle increases to $25^{\circ}$.
\end{abstract}

\section{Introduction}

A circular polarizer is utilized to convert linear polarization (LP) waves to circular polarization (CP) waves. It is very important in many applications when the diversity of polarization is desired. Recently, a variety of polarization converters has already been designed [1, 2]. Polarization and electromagnetic properties in artificial multiform helix structures are investigated in [3]. A novel metamaterial with double-helical wire structures has been proposed in [4]. The operation band of the metamaterial is $50 \%$ broader than that of the single-helical one, while the extinction rations are considerably lower. Another multihelical nanowire-based structure can operate from 0.49 to $1.34 \mu \mathrm{m}$ while the average extinction ratio reaches $270: 1$ for four-helix. However, this structure is difficult to fabricate [5]. A new method is presented to analyze the circular polarizer based on strip grating sandwiched between multidielectric layers [6]. It could be used to get pure circular polarization points with the $3 \mathrm{~dB}$ insertion loss. With a high purity of $99 \%$, a dualband polarization converter based on two concentric rings is proposed [7]. Its total bandwidth is up to $9.9 \%$ while the insertion loss increases to $5.1 \mathrm{~dB}$.

Designing polarizer using frequency selective surfaces (FSS) is an efficient way which has been proven practically $[8,9]$. A single-layer polarizer is proposed in [10]. The $3 \mathrm{~dB}$ axial ratio (AR) bandwidth is nearly $11.75 \%$, and the insertion loss is more than $3.2 \mathrm{~dB}$. It is found that the insertion loss could be significantly reduced by adding one FSS layer [11]. A double-layer split slot ring has been designed with $11.5 \%$ of the $3 \mathrm{~dB}$ AR bandwidth and $0.34 \mathrm{~dB}$ insertion loss. Using wet etch $\mathrm{KOH}$ technology based on the same unit geometry could improve the bandwidth to $21 \%$ while the insertion loss is about $1.1 \mathrm{~dB}$ [12]. The bandwidth of the polarizer based on bisected split-ring FSS is up to 57\%, and the insertion loss is less than $1 \mathrm{~dB}$. But the air gaps between the dielectrics cause a large assembly error, which limits its application in practice [13].

In this paper, a broadband linear-to-circular polarizer is presented based on right-angled frequency selective surfaces. The $\mathrm{AR}$ lower than $3 \mathrm{~dB}$ is over the frequency range from 6.42 to $13.70 \mathrm{GHz}$. Meanwhile, the polarizer can obtain a working frequency band over the range from 6.75 to $10.75 \mathrm{GHz}$ when the incident angle increases to $25^{\circ}$.

\section{Principle of Operation}

The polarization of the plane wave is the direction of its electric field vector, which may be in a fixed orientation or might alter with time. Circular polarization has been featured by the electric field in which the two orthogonal parts have the same amplitude and a phase difference of $90^{\circ}$. 


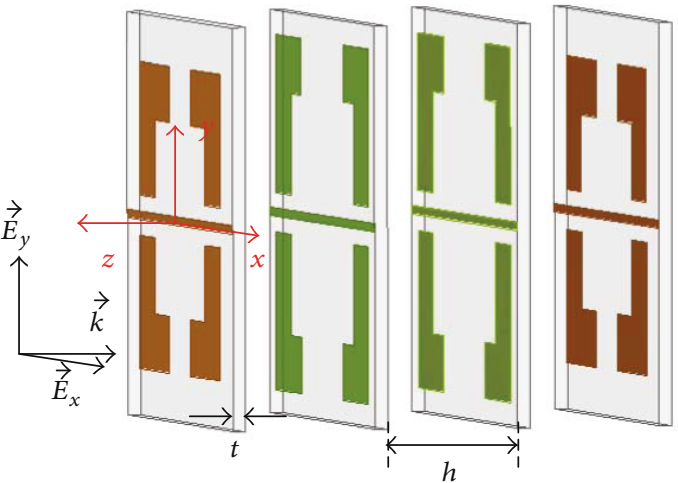

(a)

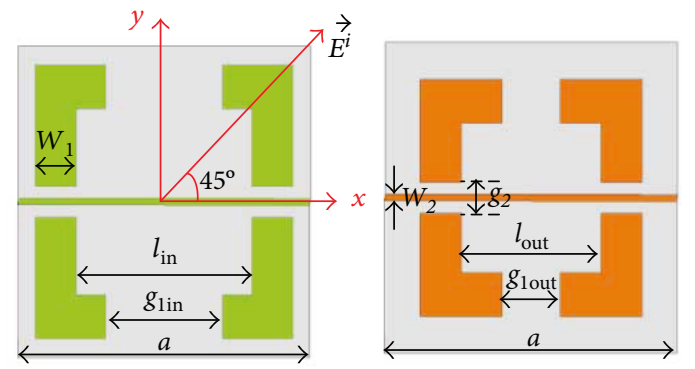

(b)

Figure 1: (a) The unit cell of the proposed circular polarizer. (b) Different geometry of the layers.

A LP wave passes through a resonator, which offers such discrepancy in the phase between two perpendicular parts. Then, a CP wave is obtained.

The proposed circular polarizer is shown in Figure 1. The system model is based on a right-angled FSS. Figure 2 shows that such a single-layer patch provides a certain phase shift, but not $90^{\circ}$ as required. So we superimpose a four-layer architecture, which achieved not only the desired phase difference but also the good transmission coefficient. Furthermore, different layers of different sizes make the performance of the proposed circular polarizer more perfect and the working bandwidth is expanded effectively.

The proposed right-angled FSS circular polarizer is used to obtain a CP exit wave from a $45^{\circ}$ title incident LP wave. The structure is composed of four dielectric layers with FSS patches which are separated by air. The size of the first layer and the forth layer is the same, and so it goes with the second layer and the third layer. Figure 1(b) presents the front view of the different layers. Each FSS patch contains four rightangled and a strip-shaped metal. The four patches are aligned along the $z$-axis with zero displacement in the $x-y$ plane. And the size of the first and forth layers is slightly smaller than the inside ones.

A LP plane wave from the $z>0$ incident can be assumed on the surface of the $x-y$ plane without generality loss. The adopted LP wave of $45^{\circ}$ tilt incident can be decomposed into two even parts in horizontal and vertical directions accordingly:

$$
\vec{E}^{i}=\vec{E}_{x}^{i}+\vec{E}_{y}^{i}=E_{0}(\widehat{\mathbf{x}}+\widehat{\mathbf{y}}) e^{j k z},
$$

where the unit vectors $\hat{x}, \hat{y}$ are parallel and perpendicular, respectively, to the metal strip. Generally, this configuration responses to a decomposition of a $\vec{E}_{x}^{i}$ and a $\vec{E}_{y}^{i}$ wave with equal phase and magnitude. If the right-angled FSS has no reflection and loss, the two parts will be completely transmitted. The circuit models of each layer FSS for $y$ and $x$ directions are shown in Figure 3. A parallel resonant circuit $L_{1}-C_{1}$ represents the right-angled shape. The capacitances $C_{x}$ and $C_{y}$ represent the effect of the fringing field concentration in the horizontal and vertical gaps, respectively, while

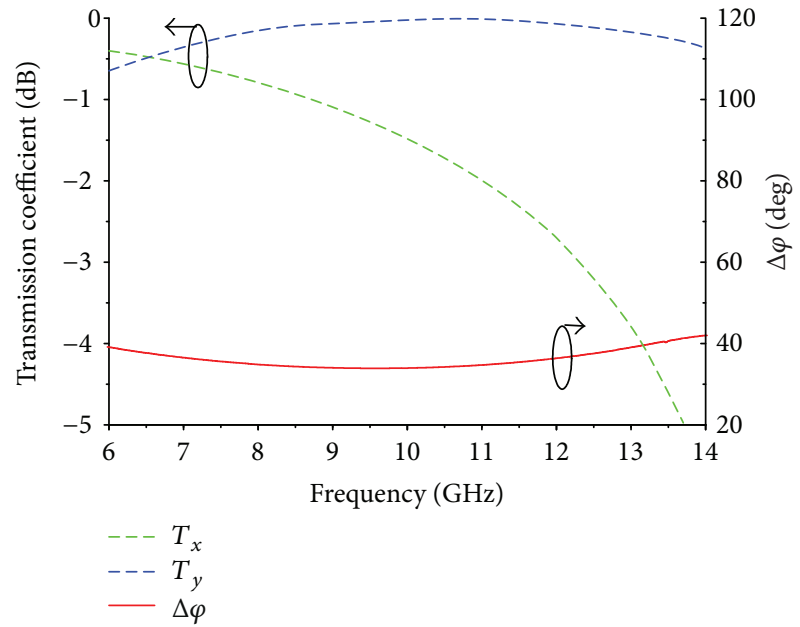

FIgURE 2: The transmission coefficients and phase difference of the single layer.

$L_{\mathrm{MS}}$ is the inductance of the metal strip in the $x$-direction. $\mathrm{TL}_{\text {sub }}$ is the transmission line that takes into account the dielectric substrate. The circuit model of the proposed polarizer is built by cascading four stages connected with the transmission lines. So it is easy to know that the polarizer plays different roles in the two incident waves. One of the two parts refers to the phase advanced by $\varphi_{x}$, while another refers to the phase retarded by the same amount of $\varphi_{y}$. This can be realized for the travel of wave via the polarizer by settling that the phase of one $x$-direction comes across an inductive path $\varphi_{x}$ because of the metal strip; nonetheless, another direction comes across a capacitive path due to the slot. Ideally, the transmitted wave can also be presented as the sum of two orthogonal linearly polarized components with equal magnitudes:

$$
\vec{E}^{t}=\vec{E}_{x}^{t}+\vec{E}_{y}^{t}=E_{0}\left(T_{x} \widehat{\mathbf{x}}+T_{y} \widehat{\mathbf{y}}\right) e^{j k z},
$$

where $T_{x}=\left|T_{x}\right| e^{j \varphi_{x}}$ and $T_{y}=\left|T_{y}\right| e^{j \varphi_{y}}$ are the transmission coefficients of $\vec{E}_{x}^{i}$ and $\vec{E}_{y}^{i}$, respectively. 


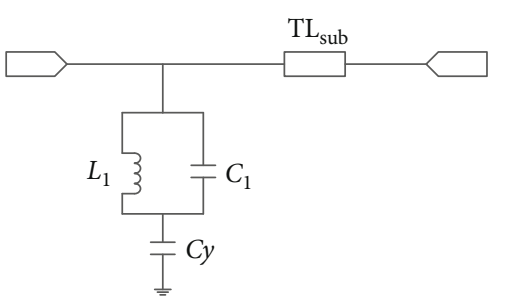

(a)

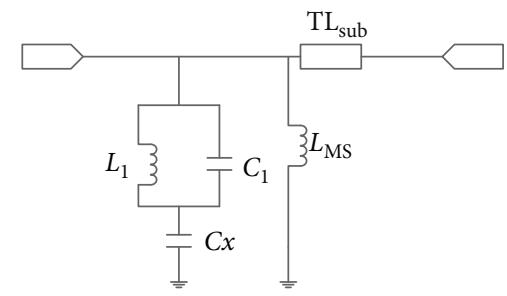

(b)

FIgURE 3: Circuit models for single FSS (a) $y$-direction and (b) $x$-direction.
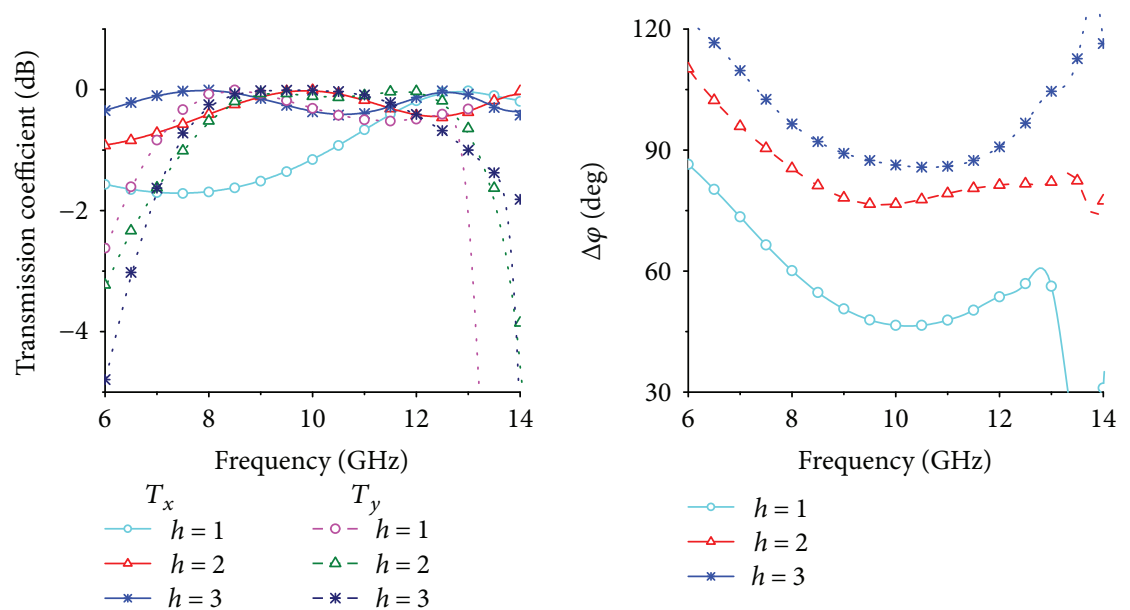

Figure 4: Simulation results of the transmitted wave for different $h$ : Transmission coefficient and phase difference.

Thus, a phase shift $\Delta \varphi=\varphi_{x}-\varphi_{y}$ appears between the two components at the output of the right-angled FSS. The parameters of the polarizer are adjusted to create $\left|T_{x}\right|=\left|T_{y}\right|$ and $\Delta \varphi= \pm 90^{\circ}$. Therefore, $90^{\circ}$ differential phase and equi-magnitude conditions are united; the exit wave from the polarizer could be an excellently $\mathrm{CP}$ wave.

\section{Optimization Design and Manufacturing}

3.1. Unit Cell Design. To acquire the preferable design parameters, the right-angled FSS centering at $10 \mathrm{GHz}$ is designed on a $0.5 \mathrm{~mm}$ thick substrate with a relative permittivity of 2.2. A unit cell of the proposed polarizer basically consists of four layers separated by a distance of $h$. With the $h$ increasing from $1 \mathrm{~mm}$ to $3 \mathrm{~mm}$, the phase difference expands obviously as shown in Figure 4 . On the other hand, the electromagnetic waves reduce seriously if the distance is down to $1 \mathrm{~mm}$.

Then, the width of the metal strip throughout the patch, $w_{2}$, is set to $0.3,0.6$, and $0.9 \mathrm{~mm}$. Based on Figure 5, both the amplitude and phase shift deteriorate at higher frequencies as the strip widens while the $\Delta \varphi$ has a steady growth at lower frequencies. Finally, the optimized geometrical parameters are as follows: $h=2 \mathrm{~mm}, a=15 \mathrm{~mm}, l_{\text {out }}=7.2 \mathrm{~mm}$, $w_{1}=2.1 \mathrm{~mm}, g_{\text {lout }}=3 \mathrm{~mm}, g_{2}=1.5 \mathrm{~mm}$, and $w_{2}=0.3 \mathrm{~mm}$. For the two middle layers, the metallic patches are much bigger as $l_{\text {in }}=9 \mathrm{~mm}$ and $g_{\text {1in }}=6 \mathrm{~mm}$; other settings are the same as the outer ones.

3.2. Experiment Results. For experiments, the polarizer each layer consisting of $13 \times 13$ elements is fabricated and tested. The metallic layers are modeled as a $0.05 \mathrm{~mm}$ copper film with an electric conductivity $\sigma=5.8 \times 10^{7} \mathrm{~S} / \mathrm{m}$. The substrate is selected as Arlon Diclad 880 with a relative permittivity of 2.2 and a dielectric loss tangent of 0.009 , whose thickness $t=0.5 \mathrm{~mm}$. The setup used to test the sample is shown in Figure 6. It is carried out in a microwave anechoic chamber in order to reduce the jamming. A vector network analyzer (VNA) is applied in measuring the polarizer, which is in connection with two standard linearly polarized horn antennas radiating electromagnetic waves in the broadband of $7-16 \mathrm{GHz}$. The two horn antennas are aligned for maximum reception, and the sample polarizer is fixed vertically on rotary stage $\mathrm{I}$ at the center of horn antennas. The placement of the sample polarizer shown in Figure 6(a) ensures that the incident LPW is oriented at $\psi=45^{\circ}$ to the $x$-axis of the polarizer. The amplitudes and phases of the transmission coefficients can be measured by the VNA. 

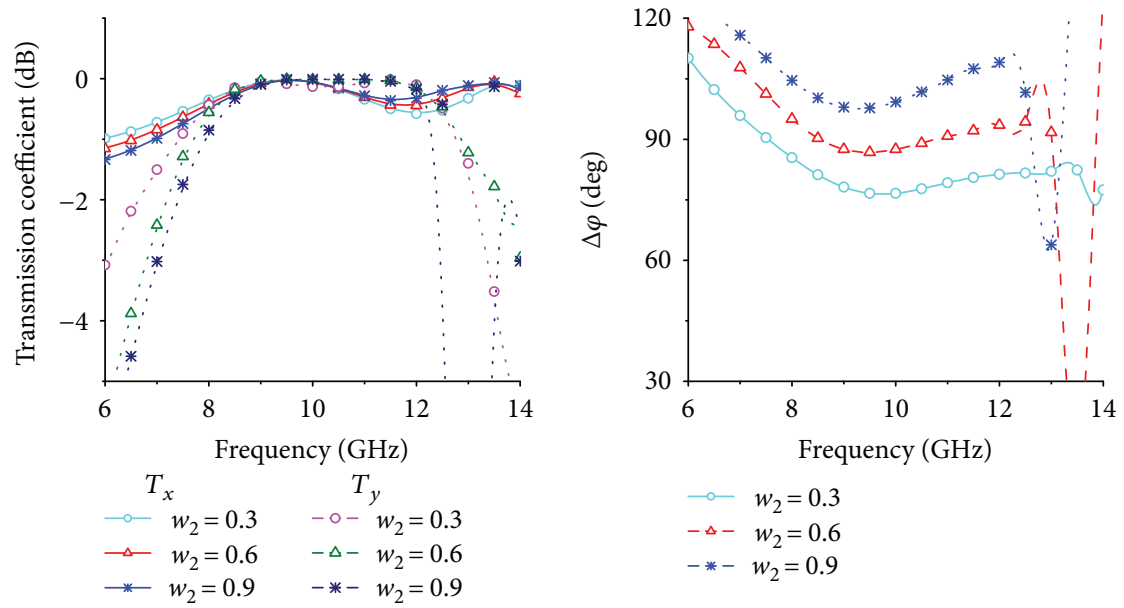

FIgURE 5: Simulation results of the transmitted wave for different $w_{2}$ : Transmission coefficient and phase difference.

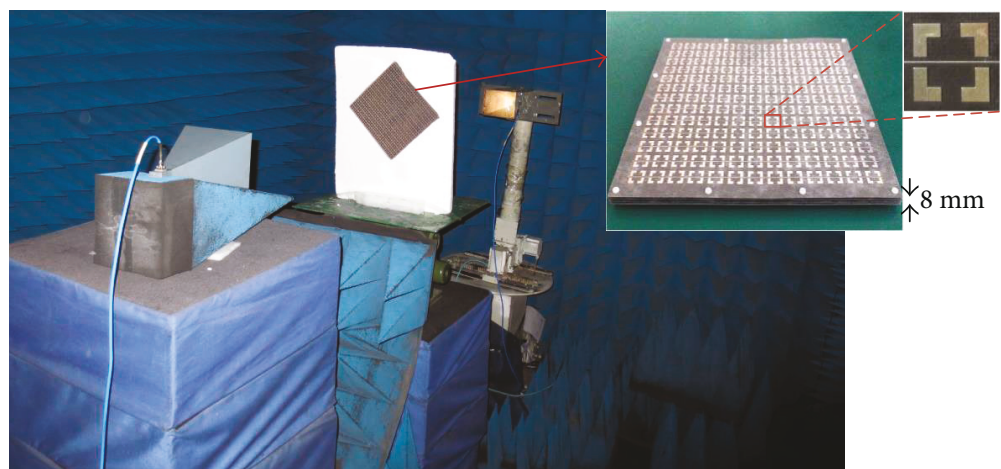

(a)

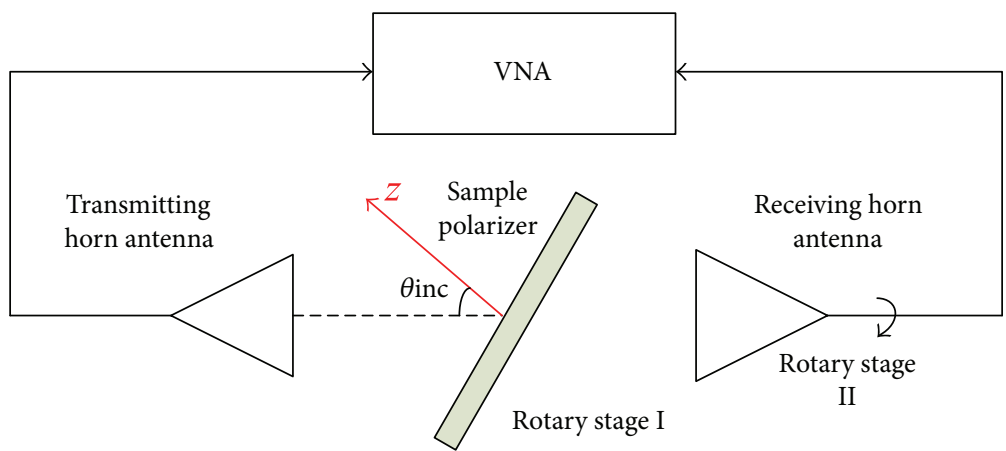

(b)

Figure 6: (a) Sample polarizer and test scenario. (b) Diagram of the setup.

The inclination angle $\tau$ of the ellipse can be generated by (5).

$$
\begin{aligned}
\mathrm{AR} & =\left[\frac{1 / a \cos ^{2} \tau+\sin 2 \tau \cos \Delta \varphi+a \sin ^{2} \tau}{1 / a \sin ^{2} \tau-\sin 2 \tau \cos \Delta \varphi+a \cos ^{2} \tau}\right], \\
a & =\frac{\left|\vec{E}_{x}^{t}\right|}{\left|\vec{E}_{y}^{t}\right|},
\end{aligned}
$$

$\tan 2 \tau=\frac{2 a}{1-a} \cos \Delta \varphi$
The magnitudes of the transmission coefficients $T_{x}$ and $T_{y}$ are shown in Figure $7(\mathrm{a})$, as well as the reflection coefficients $\left|\Gamma_{x}\right|$ and $\left|\Gamma_{y}\right|$. The $-1 \mathrm{~dB}$ bandwidth of the two transmission coefficients is $76.91 \%$, from 6.13 to $13.16 \mathrm{GHz}$. Figure $7(\mathrm{~b})$ shows the phase difference $\Delta \varphi$ and the $A R$ of the transmitted wave that is lower than $3 \mathrm{~dB}$, which can be obtained in the band of frequency from 6.42 to $13.70 \mathrm{GHz}$. A rational agreement between the simulated and measured results is detected. Therefore, as for these findings, the polarizer tends to offer 


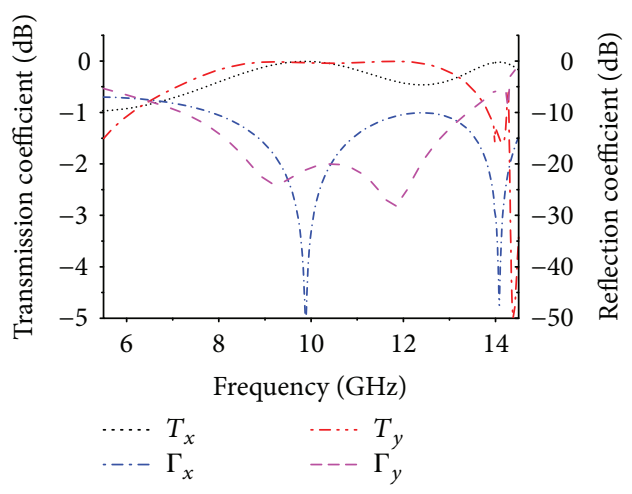

(a)

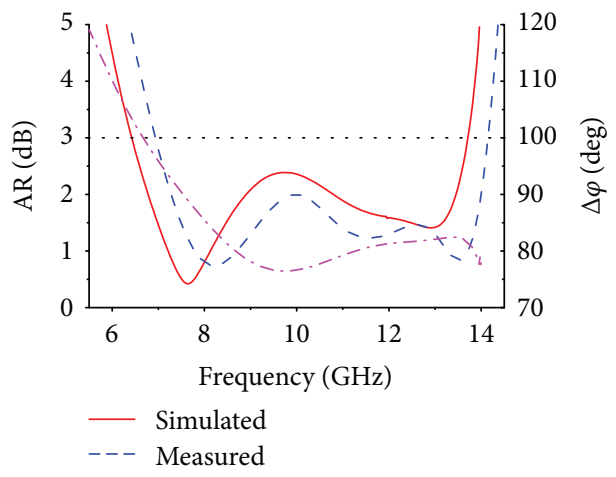

(b)

Figure 7: Simulated and measured results for normal incidence. (a) Transmission and reflection coefficients. (b) Phase difference and AR of the transmitted wave.

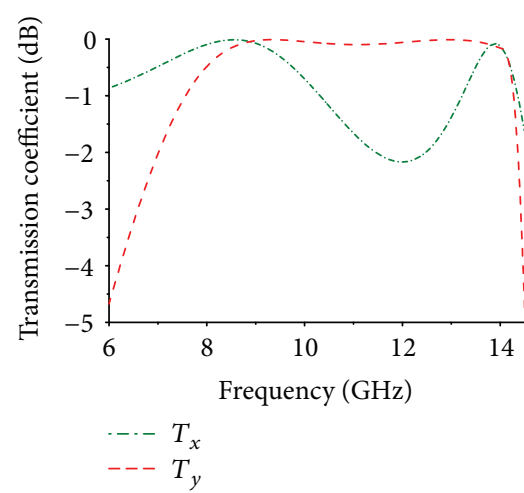

(a)

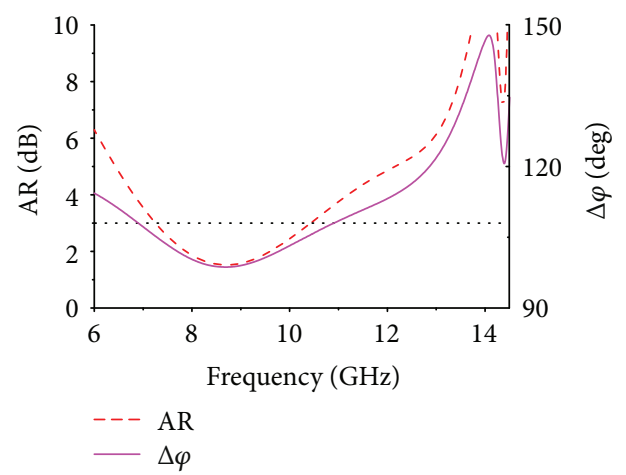

(b)

FIGURE 8: Simulated results of the polarizer with four the same layers.

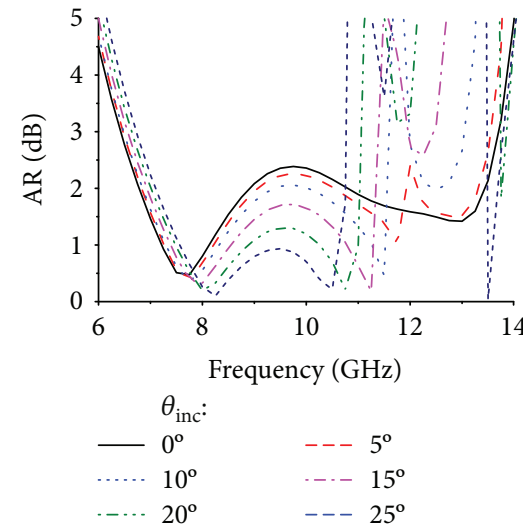

(a)

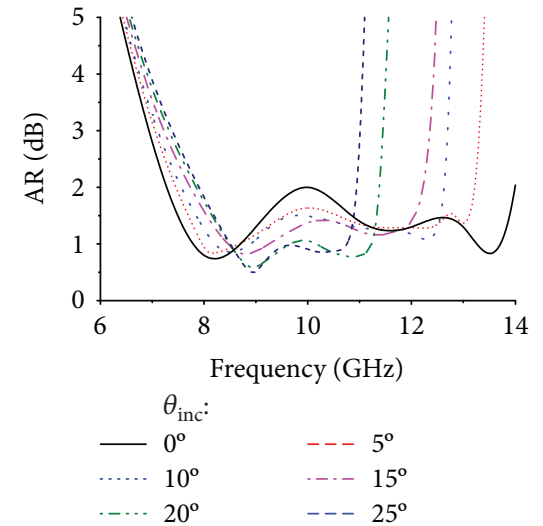

(b)

FIgURE 9: AR varied as the incident angle. (a) Simulated AR. (b) Measured AR.

an output signal with good quality. It is worth noting that the insertion losses of the polarizer are fairly minimal as there is a lack of high loss tangent strut between every layer, which is found to be only $0.06 \mathrm{~dB}$ at the center operating frequency.

\section{Discussion}

4.1. Comparisons. As displayed in Figure 1, the dimension of the first layer is consistent with the fourth one and smaller than the two ones in the middle slightly. What would happen 
without such subtle adjustment? Figure 8 gives the artificial results when the four layers of the same FSS patched. Compared with Figure 7 , it is clearly that the above patter has a great influence on the phase shift. The improper shift directly leads to a poor axis ratio, with only $36 \%$ of the $3 \mathrm{~dB}$ bandwidth. Meanwhile, the transmission coefficients also deteriorate since the loss is larger and bandwidth is smaller.

4.2. Oblique Incidence. The transmission features of any periodic structure would be varied according to a different incidence angle $\theta_{\text {inc }}$, no matter it is finite or infinite. So we have been assessed for different angles of oblique incidence. Figure 9 presents the results that the AR worsens due to high values of $\theta_{\text {inc }}$. The measured results coincide with the simulated results reasonably. It is interesting that such deterioration proves to be more pronounced for higher frequencies whereas lower frequencies are better. The $3 \mathrm{~dB} A R$ bandwidth reduces with the deteriorative loss of transmission and $\Delta \varphi$ as the incident angle increased. Furthermore, around the central frequency of $10 \mathrm{GHz}(8-10 \mathrm{GHz})$, the AR is lower and the polarizer is less sensitive to the changes of the incidence angle.

\section{Conclusion}

The right-angled frequency selective surface has been proposed as a circular polarizer in this paper. The investigated results show that the $3 \mathrm{~dB}$ axial ratio for the proposed polarizer is in excess of $70 \%$ over a wide angular bandwidth at normal incidence. The polarizer seems less sensitive to the incidence angle variations near the center frequency of $10 \mathrm{GHz}(8-10 \mathrm{GHz})$. Furthermore, the insertion loss is only $0.06 \mathrm{~dB}$ omitting the strut. A reasonable agreement between simulated and measure results is obtained.

\section{Conflicts of Interest}

The authors declare that they have no competing interests.

\section{Acknowledgments}

This work was supported in part by the National Natural Science Foundation of China (nos. 61271416 and 61301093), the Fundamental Research Funds for the Central Universities (no. 3102014KYJD027), and NPU Foundation for Fundamental Research (no. JCY20130132). The authors would like to thank Wei Kun and Xu Rui for their kind assistance with the test.

\section{References}

[1] D. Lerner, "A wave polarization converter for circular polarization," IEEE Transactions on Antennas and Propagation, vol. 13, no. 1, pp. 3-7, 1965.

[2] L. Young, L. Robinson, and C. Hacking, "Meander-line polarizer," IEEE Transactions on Antennas and Propagation, vol. 21, no. 3, pp. 376-378, 1973.

[3] N. Wongkasem, "Circular polarizer by multi-form helices," in 2014 22nd International Conference on Software,
Telecommunications and Computer Networks (SoftCOM), pp. 37-40, Split, Croatia, 2015.

[4] Z. Y. Yang, M. Zhao, and Y. F. Lu, "Similar structures, different characteristics: optical performances of circular polarizers with single- and double-helical metamaterials," Journal of Lightwave Technology, vol. 28, no. 21, pp. 3055-3061, 2010.

[5] Z. Yang, M. Zhao, and P. Lu, "How to improve the signal-tonoise ratio for circular polarizers consisting of helical metamaterials?," Optics Express, vol. 19, no. 5, pp. 4255-4260, 2011.

[6] W.-H. Fang, F. Nian, and L. Zhang, "Analysis of scattering characteristics for strip grating sandwiched between multidielectric layers under oblique incidence and its applications in circular polarizers," in 2010 International Conference on Microwave and Millimeter Wave Technology, pp. 1156-1159, Chengdu, China, 2010.

[7] Y. Ranga, S. G. Hay, and T. S. Bird, “An anisotropic impedance surface for dual-band linear-to-circular transmission polarization convertor," in 2013 International Workshop on Antenna Technology (iWAT), pp. 47-50, Karlsruhe, Germany, 2013.

[8] Y. Li, J. Zhang, and S. Qu, "Achieving wide-band linearto-circular polarization conversion using ultra-thin bilayered metasurfaces," Journal of Applied Physics, vol. 117, pp. 044501-1-044501-7, 2015.

[9] D. Zarifi, M. Soleimani, and V. Nayyeri, "Dual- and multiband chiral metamaterial structures with strong optical activity and negative refraction index," IEEE Antennas and Wireless Propagation Letters, vol. 11, pp. 334-337, 2012.

[10] M. Euler, V. Fusco, R. Cahill, and R. Dickie, "325 GHz single layer sub-millimeter wave FSS based split slot ring linear to circular polarization convertor," IEEE Transactions on Antennas and Propagation, vol. 58, no. 7, pp. 2457-2459, 2010.

[11] M. Euler, V. Fusco, and R. Cahill, "Comparison of frequencyselective screen-based linear to circular split-ring polarisation convertors," IET Microwaves Antennas \& Propagation, vol. 4, no. 11, pp. 1764-1772, 2010.

[12] M. Euler, V. Fusco, and R. Dickie, "Sub-mm wet etched linear to circular polarization FSS based polarization converters," IEEE Transactions on Antennas and Propagation, vol. 59, no. 8, pp. 3103-3106, 2011.

[13] L. Martinez-Lopez, J. Rodriguez-Cuevas, J. I. Martinez-Lopez, and A. E. Martynyuk, "A multilayer circular polarizer based on bisected split-ring frequency selective surfaces," IEEE Antennas and Wireless Propagation Letters, vol. 13, pp. 153-156, 2014. 


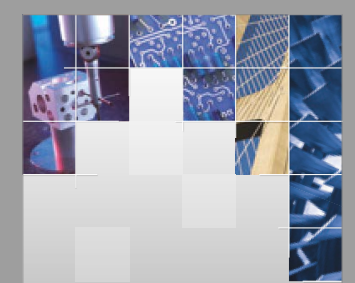

\section{Enfincering}
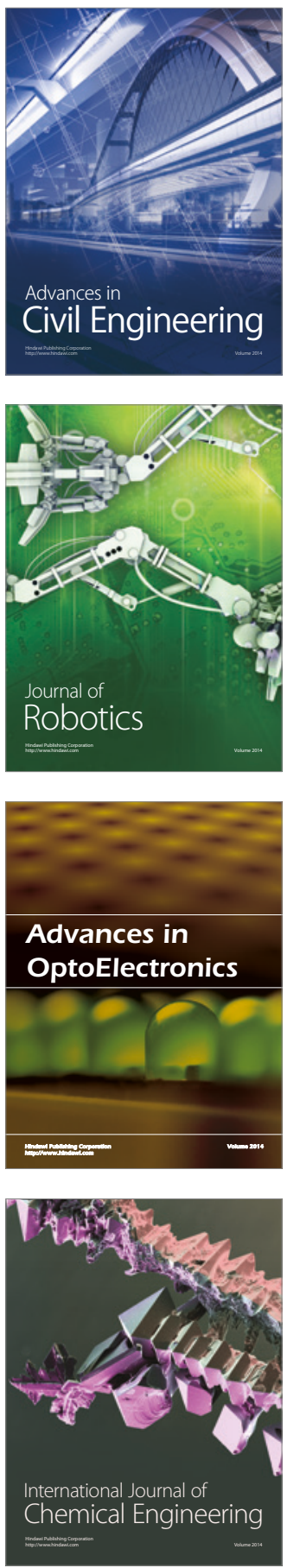

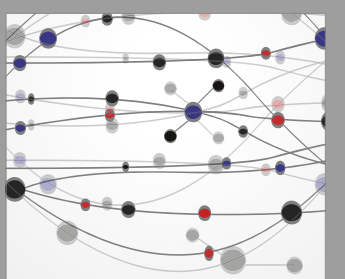

The Scientific World Journal

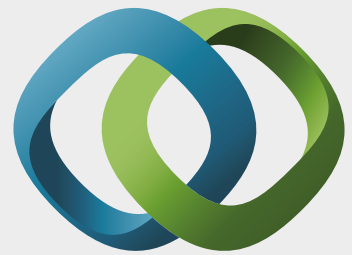

\section{Hindawi}

Submit your manuscripts at

https://www.hindawi.com
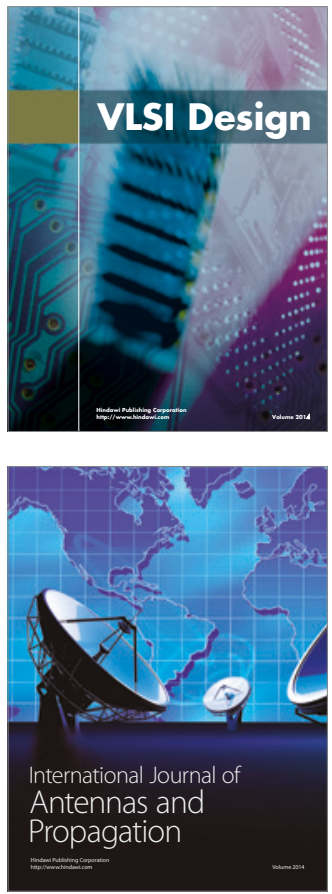

\section{Rotating}

Machinery
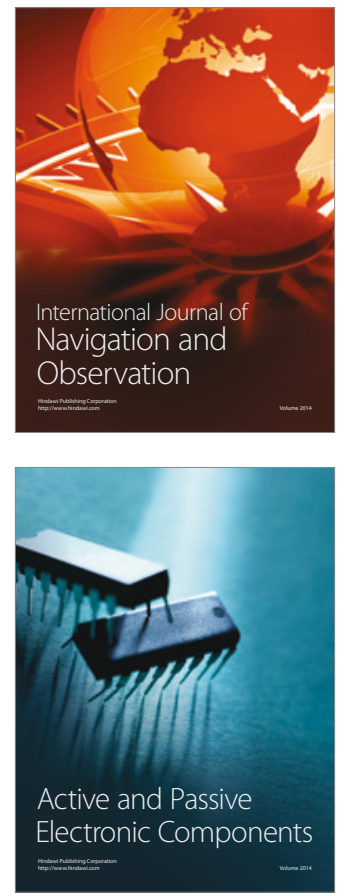
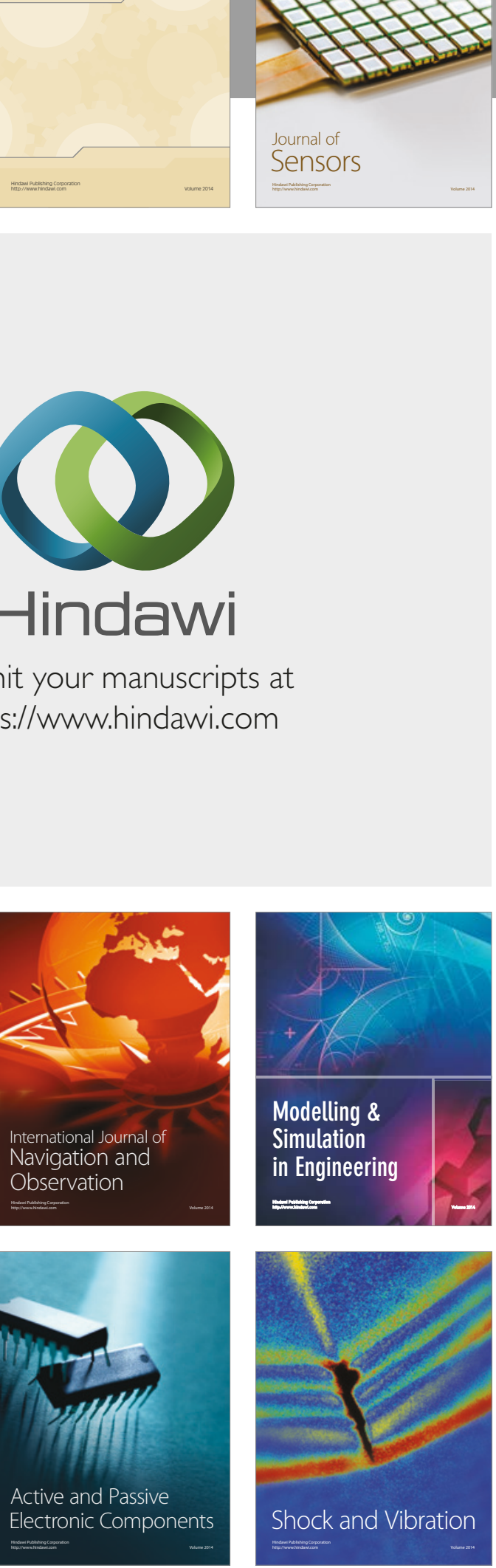
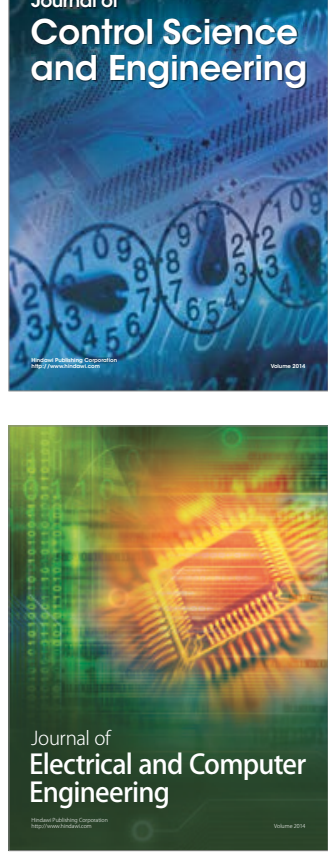

Distributed

Journal of

Control Science

and Engineering
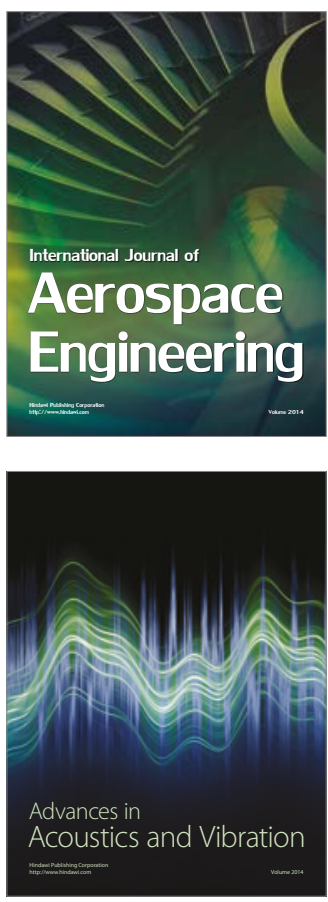

Sensor Networks 